\title{
Semiconductor-based narrow-line and high-brilliance 193-nm laser system for industrial applications
}

D. Opalevs, M. Scholz, J. Stuhler, C. Gilfert, L. J. Liu, et al.

D. Opalevs, M. Scholz, J. Stuhler, C. Gilfert, L. J. Liu, X. Y. Wang, A. Vetter, R. Kirner, T. Scharf, W. Noell, C. Rockstuhl, R. K. Li, C. T. Chen, R. Voelkel, P. Leisching, "Semiconductor-based narrow-line and high-brilliance 193-nm laser system for industrial applications," Proc. SPIE 10511, Solid State Lasers XXVII: Technology and Devices, 105112C (15 February 2018); doi: $10.1117 / 12.2290288$

SPIE. Event: SPIE LASE, 2018, San Francisco, California, United States 


\title{
Semiconductor-based narrow-line and high-brilliance $193 \mathrm{~nm}$ laser system for industrial applications
}

\author{
D. Opalevs ${ }^{1}$, M. Scholz ${ }^{1}$, J. Stuhler ${ }^{1}$, C. Gilfert ${ }^{1}$, L.J. Liu ${ }^{2}$, X.Y. Wang ${ }^{2}$, A. Vetter ${ }^{3,4}$, R. Kirner ${ }^{4}$, \\ T. Scharf ${ }^{5}$, W. Noell ${ }^{4}$, C. Rockstuhl ${ }^{3,6}$, R.K. Li ${ }^{2}$, C.T.Chen ${ }^{2}$, R. Voelkel $^{4}$ and P. Leisching ${ }^{*}$ \\ ${ }^{1}$ TOPTICA Photonics AG, Lochhamer Schlag 19, 82166 Gräfelfing, Germany \\ ${ }^{2}$ Beijing Center for Crystal Growth and Development, Key Laboratory of Functional Crystals and \\ Laser Technology, Technical Institute of Physics and Chemistry, Chinese Academy of Sciences, \\ Beijing 100190, China \\ ${ }^{3}$ Institute of Nanotechnology (INT), Karlsruhe Institute of Technology, \\ 76344 Eggenstein-Leopoldshafen, Germany \\ ${ }^{4}$ SUSS MicroOptics SA, Rouges-Terres 61, 2068 Hauterive, Switzerland \\ ${ }^{5}$ Nanophotonics and Metrology Laboratory, École Polytechnique Fédérale de Lausanne (EPFL), \\ 1015 Lausanne, Switzerland \\ ${ }^{6}$ Institute of Theoretical Solid State Physics (TFP), Karlsruhe Institute of Technology, \\ 76131 Karlsruhe, Germany
}

\begin{abstract}
We present a novel industrial-grade prototype version of a continuous-wave $193 \mathrm{~nm}$ laser system entirely based on solid state pump laser technology. Deep-ultraviolet emission is realized by frequency-quadrupling an amplified diode laser and up to $20 \mathrm{~mW}$ of optical power were generated using the nonlinear crystal KBBF. We demonstrate the lifetime of the laser system for different output power levels and environmental conditions. The high stability of our setup was proven in $>500 \mathrm{~h}$ measurements on a single spot, a crystal shifter multiplies the lifetime to match industrial requirements. This laser improves the relative intensity noise, brilliance, wall-plug efficiency and maintenance cost significantly. We discuss first lithographic experiments making use of this improvement in photon efficiency.
\end{abstract}

Keywords: Deep-ultraviolet, frequency-doubled diode laser, narrow band, nonlinear crystal, KBBF, metrology, lithography, spectroscopy

\section{INTRODUCTION}

For modern industrial applications in metrology and lithography in the deep-ultraviolet (DUV) spectral region, the pulsed $193 \mathrm{~nm}$ excimer laser system is still the state-of-the-art tool [1-4]. However, these systems have a low wall-plug efficiency and low photon efficiency, cumbersome usability and require an expensive maintenance program. Replacing these lasers by all semiconductor-based systems offers significant cost reduction and operating benefits for industrial and research spectroscopic applications, e.g. angle-resolved photoemission spectroscopy (ARPES) [5]. First experiments have already demonstrated the principal feasibility of this compact and high wall-plug efficiency technology on a scientific level. However the lifetime was limited to below $100 \mathrm{~h}$ [6-8].

In the first section of this paper we explain the experimental setup of the industrial prototype version of the laser system and present results on the optical performance of the narrow-line $193 \mathrm{~nm}$ laser system realized by frequency-quadrupling an amplified diode laser. The second section discusses the high mechanical and long-term stability of this compact laser system and presents measurements on a single optical spot. To match industrial lifetime requirements, a crystal shifter multiplies the per-spot lifetime. We also discuss the service concept and the lifetime of the laser system for different output power levels and environmental conditions. The final section highlights the successful integration of the laser source in a mask aligner setup and shows first proximity lithography experiments with submicron resolution to indicate the potential in mask aligner lithography. The final section comprises a conclusion and further outlook for the reduction of size and the power scaling of the laser system to enable wider use in more applications.

*patrick.leisching@toptica.com; phone: +49-(0)89-85837-0; fax: +49-(0)89-85837-200; www.toptica.com

Solid State Lasers XXVII: Technology and Devices, edited by W. Andrew Clarkson, Ramesh K. Shori, Proc. of SPIE Vol. 10511, 105112C · (c) 2018 SPIE · CCC code: 0277-786X/18/\$18 · doi: 10.1117/12.2290288 


\section{LASER DESIGN AND EXPERIMENTAL SETUP}

The quest for DUV continuous-wave (CW) laser systems is hampered by fundamental laser physics: The shorter the wavelength, the more challenging is the fulfillment of the lasing criterion, see e.g. [9]. Only with pulsed excimer and nitrogen lasers, wavelengths below $375 \mathrm{~nm}$ are reachable by direct laser transitions. However, pulsed lasers generally lead to higher degradation of optical materials and coatings in the DUV, see e.g. [10, 11]. To enable CW emission, laser sources in the near-infrared or visible spectral range are frequency-converted in nonlinear crystals to generate light in the ultraviolet and deep-ultraviolet spectrum. Considering the cost and complexity of these laser systems, second-harmonic processes from a single fundamental source are generally favored over mixing processes between different pump lasers. The principle setup of the frequency-quadrupled laser system is shown in Fig. 1, the technical details are explained in Ref. [6-8]. The seed laser is a TOPTICA DL pro laser system at $772 \mathrm{~nm}$ and a linewidth of less than $50 \mathrm{kHz}$. This laser is amplified with a tapered amplifier to about $3 \mathrm{~W}$ optical output power. Optical isolators protect seed and amplifier from detrimental back-reflections. There are two enhancement cavities for efficient frequency doubling. The crystal material for the first nonlinear process is lithium triborate (LBO). The crystal material for the second frequency-doubling stage is potassium fluoro-beryllo-borate (KBBF) [12-14].

To increase the lifetime of the DUV part of the system, the second cavity is filled with a protective gas mixture with a leakage rate of less than $10^{-9} \mathrm{mbar} 1 / \mathrm{s}$. A similar concept is used in the TopWave technology of TOPTICA's CW 266 nm laser system with more than $300 \mathrm{~mW}$ output power, see e.g. [15].

The laser head is incorporated into an aluminum OEM package and electronically driven by the digital controller DLC pro system, see e.g. [16]. A picture of the system mounted into a lithography development test-bench in the clean room environment at SUSS is shown on the right hand side of Fig. 1.

A careful characterization of the electrical and optical parameters of the prototype system has been performed. With an electrical input power of $60 \mathrm{~W}$, we achieved maximum optical output powers of $3 \mathrm{~W}$ at $772 \mathrm{~nm}, 1.7 \mathrm{~W}$ at $386 \mathrm{~nm}$ and up to $20 \mathrm{~mW}$ at $193 \mathrm{~nm}$. The linewidth at $193 \mathrm{~nm}$ is at the order of $100 \mathrm{kHz}$, delivering high-coherence photons for metrology and spectroscopy. The relative intensity noise of $0.22 \%(10 \mathrm{~Hz}$ to $10 \mathrm{MHz})$ offers a major improvement for noise-sensitive applications as table-top excimer laser sources show typical pulse-to-pulse power fluctuations of 1-2\%. The volume of the system shown in Fig. 1 is about $0.03 \mathrm{~m}^{3}$, by far smaller than conventional DUV laser systems. Using spatial filtering $\mathrm{M}^{2}<1.2$ can be easily achieved. The wall-plug efficiency electron to laser photon is comparable to excimer lasers, however due to the spectral and spatial brilliance a large improvement of electron to photon efficiency on the mask is expected. No water cooling with e.g. a chiller is required.
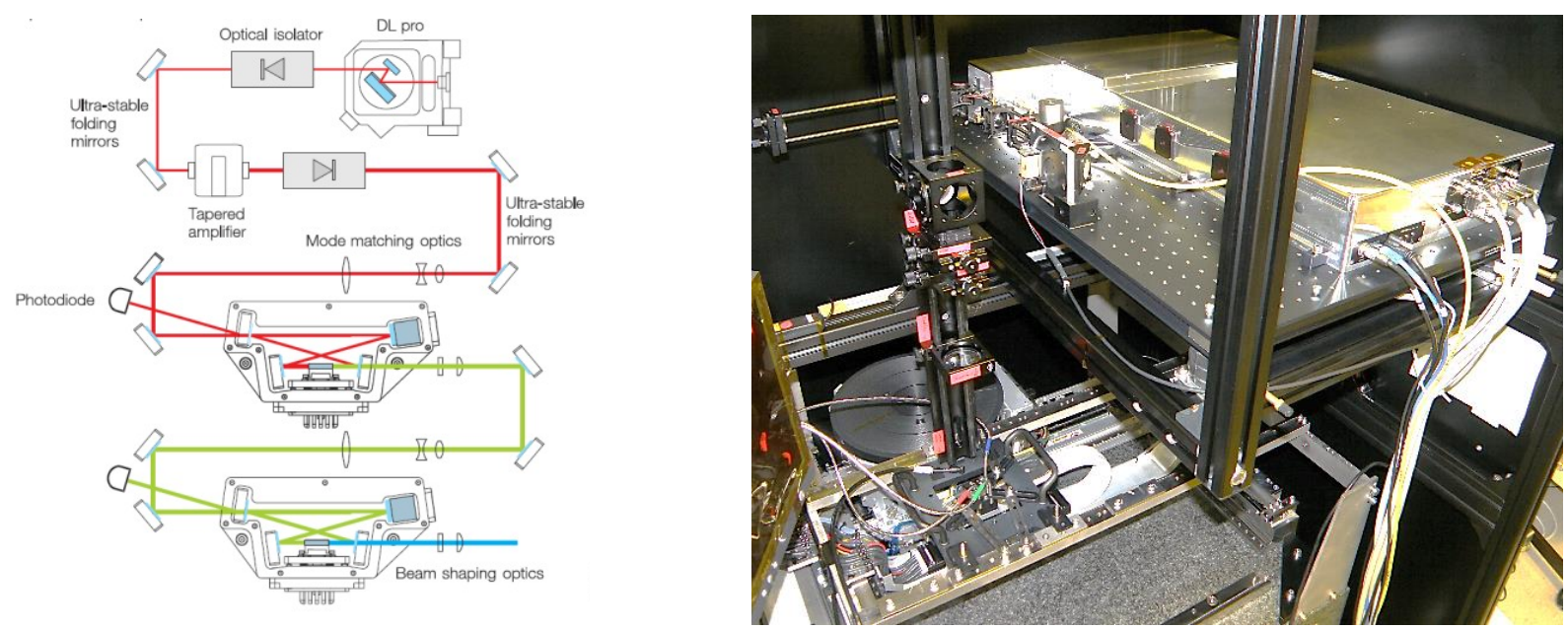

Figure 1: The picture on the left shows the schematic set-up of a frequency-quadrupled laser system. The picture on the right depicts the laser system integrated in a test-bench lithography system at SUSS.

In the past, the generation of CW light relying on diode and solid-state lasers for wavelengths below $200 \mathrm{~nm}$ has been practically inhibited by the unavailability of suitable materials, i.e., nonlinear crystals with frequency-conversion properties in the desired wavelength range. The introduction of high-quality KBBF crystals has just been closing this gap. The trade-off of this crystal is the mechanical mount, as it needs to be bonded to a $\mathrm{CaF}_{2}$ prism to couple the pump 
and laser converted light in and out, see Fig. 2. To cope with the $\mathrm{KBBF} / \mathrm{CaF}_{2}$ bonding interface degradation, additionally a crystal shifter is integrated in the second enhancement cavity.
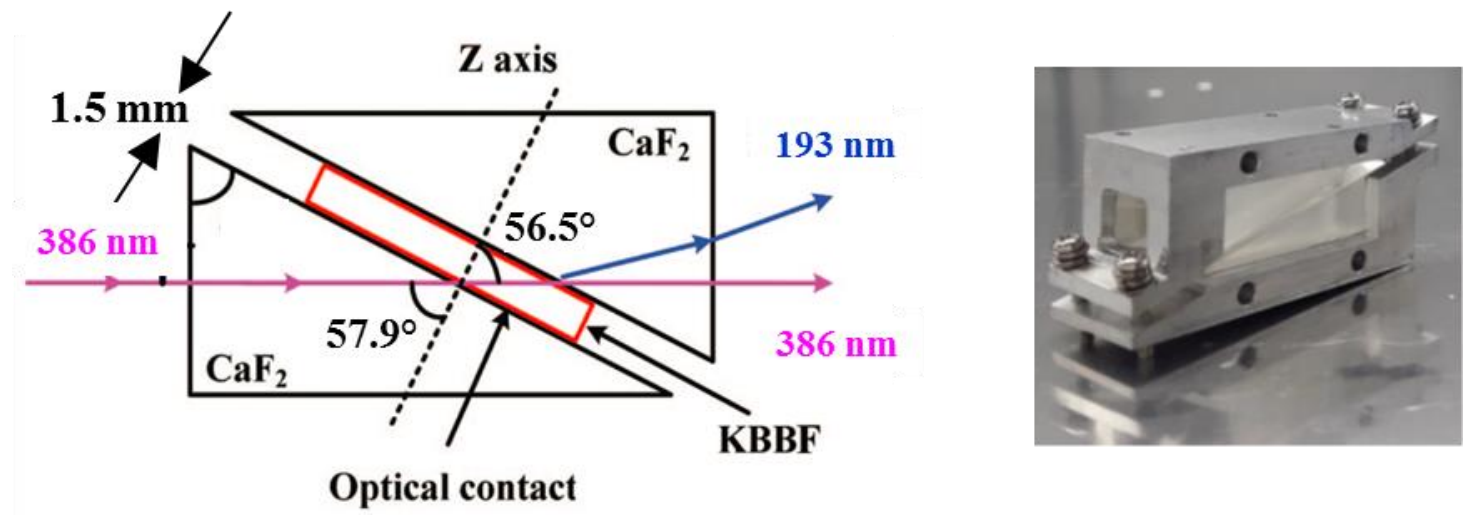

Figure 2: Left side: schematic view of the mechanical mount for the KBBF crystal between two $\mathrm{CaF}_{2}$ prisms, optimized for frequency-doubling from $386 \mathrm{~nm}$ to $193 \mathrm{~nm}$. Right side: picture of the mechanical mount for the prism-coupled KBBF crystal.

The schematic set-up is shown on the left hand side: the crystals of 1-2 mm thickness are first polished and then bonded to $\mathrm{CaF}_{2}$ prisms. This complex optical subsystem has to be mechanically mounted inside the second enhancement cavity. The crystal shifter can translate the optical subsystem to more than 40 different positions with a reproducibility of $+/-1$ $\mu \mathrm{m}$ in operation. An OEM-grade automated crystal shift results in less than $+/-50 \mu \mathrm{m}$ deviation in $1 \mathrm{~m}$ distance from the laser.

\section{LIFETIME \& ENVIRONMENTAL MEASUREMENTS}

The first experiments to achieve CW laser radiation around $193 \mathrm{~nm}$ with KBBF crystals were based on TOPTICA's scientific laser systems without crystal optics shifter [6-8]. In scientific DUV semiconductor based CW systems, the lifetime in the DUV is generally limited by the degradation of the surface and bulk volume of the optical parts of the system.

This limitation is addressed by crystal and/or optical component shifters to move the critical optical elements through the laser beam after a certain number of operating hours, see e.g. TOPTICA's TopWave OEM system at $266 \mathrm{~nm}$ [15]. The lifetime of the newly developed industrial grade $193 \mathrm{~nm}$ DUV system with shifting options was investigated for different output powers and at different temperatures, see Fig. 3.
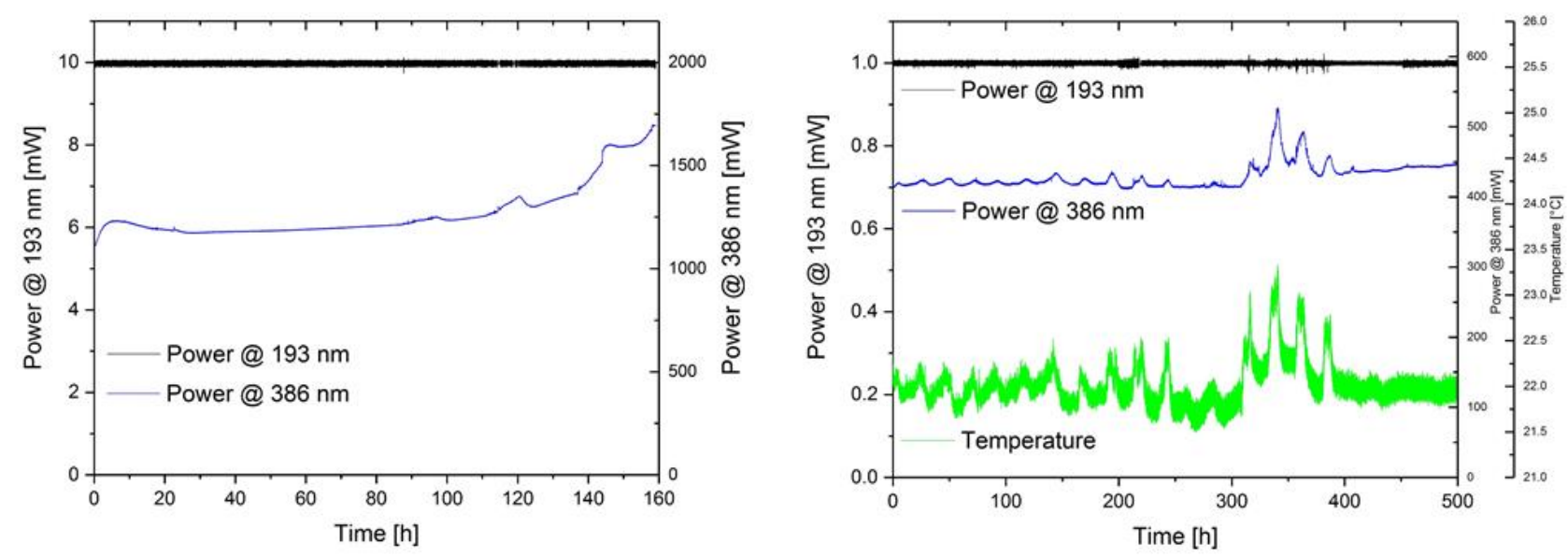

Figure 3: Time dependence of the actively stabilized output power for $10 \mathrm{~mW}$ (left) and for $1 \mathrm{~mW}$ (right). We estimate typical lifetimes without optics shifters to be up to $200 \mathrm{~h}$ for $10 \mathrm{~mW}$ and more than $500 \mathrm{~h}$ for $1 \mathrm{~mW}$ output power. The build-in shifter for the prism-coupled KBBF device multiplies these lifetimes. 
On the left hand side of Fig. 3, an output power of $10 \mathrm{~mW}$ at $193 \mathrm{~nm}$ was chosen to investigate the lifetime for higher optical output powers. The output power is actively stabilized via the pump laser power at $772 \mathrm{~nm}$ and hence the power at $386 \mathrm{~nm}$ for the frequency-doubling stage to $193 \mathrm{~nm}$ ("PowerLock" function). Even at $10 \mathrm{~mW}$, a lifetime of up to $200 \mathrm{~h}$ can be achieved before the regulated power at $386 \mathrm{~nm}$ reaches its maximum of $1.7 \mathrm{~W}$. Multiplied with an estimated number of 30-50 spots, a total lifetime of $5.000 \mathrm{~h}$ to $10.000 \mathrm{~h}$ is in reach. Reducing the output power to $1 \mathrm{~mW}$ (Fig. 3, right), lifetimes of more than $500 \mathrm{~h}$ per spot can be achieved. The degradation mechanism - most likely of the bonding interface between $\mathrm{CaF}_{2}$ and $\mathrm{KBBF}$ - is currently under investigation. An improvement will pave the way for even higher power laser systems.

The temperature dependence in operation is shown by the green curve on the right-hand side of Fig. 4: The power stabilization mechanism can control the output power over the typical temperature range in a semiconductor fab environment. The system can generally be operated within a temperature range from 15 to $30{ }^{\circ} \mathrm{C}$. To investigate the mechanical robustness of the system for transport, it was twice transported from Munich to Neuchâtel for measurement campaigns. The particular behavior of the spot positions was verified in both cases, no deviations from the initial positions were observed. Using the push-button "AutoAlign" function to automatically align the beam steering between diode laser, amplifier, first and second frequency-doubling stage of the system, the initial operating conditions could be restored.

Concerning a service concept for long lifetime operation, we envisage field-replaceable units such as the second cavity containing the KBBF crystal, a tapered amplifier module, a laser diode module and a first cavity module. The service interval will depend on the optical power and is different for the individual modules. The laser-diode module and the tapered amplifier can principally be combined in a compact master-oscillator power-amplifier module [19, 20].

\section{EXPERIMENTAL RESULTS LITHOGRAPHY}

The wavelength of $193 \mathrm{~nm}$ is compatible to the photolithography technology developed for the ArF excimer laser, especially the photoresist chemistry tailored to work in the specified illumination regime.

Mask-aligner lithography is a microfabrication technique used in research and production to transfer a mask pattern to a photoresist-coated substrate. Mask and wafer are arranged in close proximity or in contact to achieve full-field structuring with a modest resolution at low costs and high flexibility. The spatial resolution of the printing process in mask-aligner lithography can be improved by introducing a light source with a reduced wavelength. Today's state-ofthe-art ArF excimer lasers at $193 \mathrm{~nm}$ are operated at a pulsed regime [1-4] and are generally complex and expensive. Additionally, they require corrosive and large-volume gas mixtures at high pressures and voltages exceeding $40 \mathrm{kV}$ for high-power operation. Frequent maintenance, including gas exchange and electrode replacement, is required.

Moreover, in the DUV, feasible optical materials are restricted to crystalline calcium fluoride $\left(\mathrm{CaF}_{2}\right)$ and UV-grade fused silica [10]. The formation of color centers emerging from the high peak power levels in pulsed operation leads to a degradation in transmittance over time in such materials [11]. Thus a CW system is generally preferred.

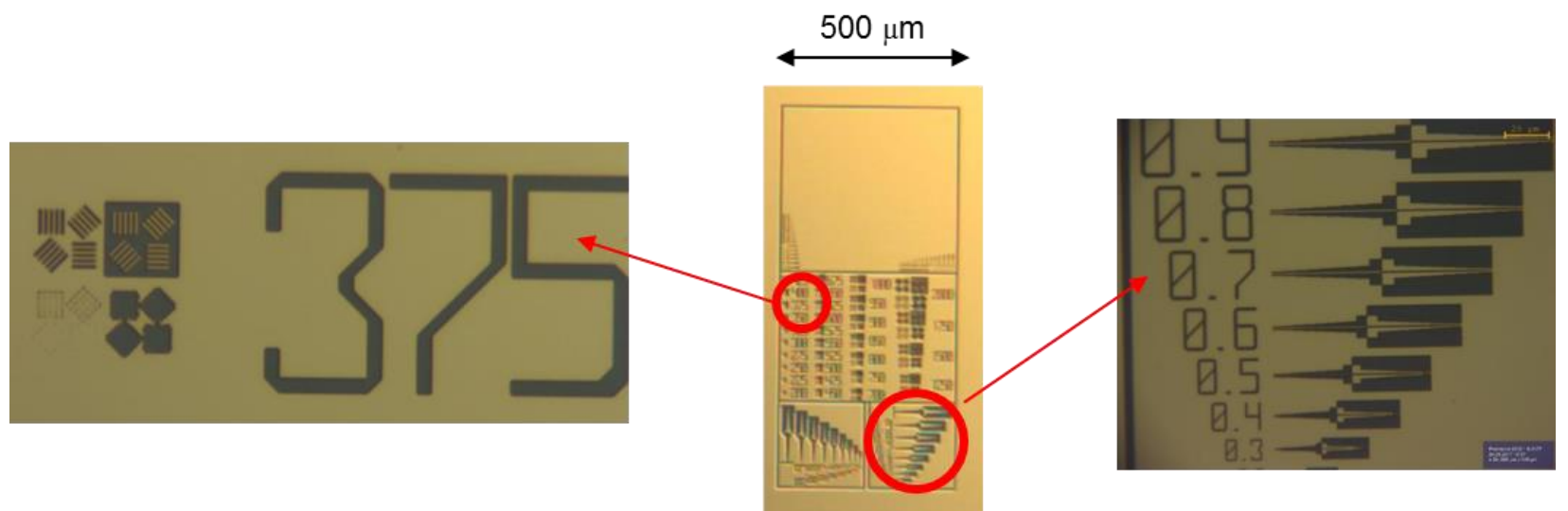

Figure 4: First experimental results of soft contact mask-aligner lithography with test-structure of $350-400 \mathrm{~nm}$. A minimum feature size of $375 \mathrm{~nm}$ is clearly visible. 
As a first proof-of-principle of the new CW laser system at $193 \mathrm{~nm}$, we present lithographic results with an amplitude mask fabricated from UV-grade fused silica, see Fig. 4. Although the proof-of-principle results are shown for print fields of $1 \mathrm{~cm}^{2}$ the technology is not limited to such small fields and can be applied to full-field mask-aligner lithography. For these first investigations an optical output power of $7 \mathrm{~mW}$ was sufficient to obtain a useful exposure time.

The high spectral and spatial brilliance of the semiconductor laser source principally enables a higher photon efficiency than for the excimer laser, thus a lower degradation of the optical materials is expected. However, for industrial lithographic applications output powers $>100 \mathrm{~mW}$ are required.

The first results in Fig. 4 demonstrate a minimum feature size smaller than $375 \mathrm{~nm}$ for amplitude masks. Using phase masks, the full potential of the new laser source will become even more prominent and a further extension using concepts of Advanced Mask Aligner Lithography (AMALITH) $[4,18]$ for resolution enhancement is feasible.

\section{CONCLUSION \& OUTLOOK}

Using the unique capabilities of the nonlinear crystal KBBF, we were able to generate DUV CW radiation around 193 $\mathrm{nm}$ using a photon efficient all-solid-state prototype laser system for industrial applications. The output wavelength was generated by two consecutive second-harmonic processes starting from a $3 \mathrm{~W}$ amplified diode laser-based pump source at $772 \mathrm{~nm}$. Compared to the gold standard ArF excimer laser, our system has a high photon efficiency with excellent photon brilliance (linewidth at $193 \mathrm{~nm}$ around $100 \mathrm{kHz}, \mathrm{M}^{2}<1.2$ ), an ultralow relative intensity noise of $0.22 \%$, and is very compact $\left(0.03 \mathrm{~m}^{3}\right)$. The characterization of our setup showed long-term operation at $1 \mathrm{~mW}$ and $10 \mathrm{~mW}$, which can be multiplied to 5.000-10.000 h using crystal shifter technology. The maximum short-term output power was $20 \mathrm{~mW}$. To increase the output power to $20-50 \mathrm{~mW}$, further research and development is needed to address the homogeneity of the $\mathrm{KBBF} / \mathrm{CaF}_{2}$ interface and improve the regularity and the lifetime of the individual crystal spots.

This technology paves the way to reach industrial standards of these formerly scientific systems, as advanced functions like "PowerLock" and "AutoAlign" will allow future push button operation. For low output power levels and on timescales of up to 5.000-10.000 h, the second enhancement cavity unit containing the KBBF crystal has to be replaced. A service concept for other field-replaceable units like the amplifier module, the diode laser and the first cavity can be easily developed and implemented.

We also demonstrated a proof-of-principle experiment the suitability of a $193 \mathrm{~nm} \mathrm{CW}$ diode laser as a light source for high-resolution mask-aligner lithography. The optical performance is comparable to prevalent excimer light sources in the DUV, while the CW operation of our light source features an improvement in ease of implementation, source stability, and thermal heat load. The necessary illumination system incorporating the DUV laser light source was discussed. In addition, in soft contact we demonstrate resist structures (lines and spaces) with minimum feature size of $375 \mathrm{~nm}$ with a binary mask.

The benefits of such CW sources for industrial applications in terms of relative intensity noise, brilliance, wall-plug efficiency, and maintenance expenditures were discussed, they offer significant advantages compared to today's ArF excimer pulsed technology. The high photon efficiency and the $\mathrm{CW}$ operation will lead to longer lifetimes for all optical components in the DUV part of the lithography system compared to excimer based systems. For metrology applications, the current power output levels are already sufficient for industrial applications due to the high brilliance of the laser system. This compact and robust laser source can be improved in the future: using a miniaturized pump laser module at $772 \mathrm{~nm}$, the size is further reduced, the robustness increased and such modules can potentially be replaced in the field $[19,20]$. Improving the bonding of the $\mathrm{KBBF}$ to the $\mathrm{CaF}_{2}$ prisms, we can significantly increase the optical output power beyond $20 \mathrm{~mW}$. Introducing such improvements, also optical lithography relying on CW DUV lasers might be within reach. However, the full potential of the laser system will become visible for modern lithography using not only amplitude but phase masks [18]. Increasing the power level to $100 \mathrm{~mW}$ and beyond will require more complex frequency mixing processes using different crystals and high-power pump fiber lasers to achieve an efficient frequency conversion process. For pulsed operation, up to $300 \mathrm{~mW}$ at $193 \mathrm{~nm}$ have already been achieved, see e.g. [21].

\section{ACKNOWLEDGEMENTS}

TOPTICA's work on this project was supported by the German Federal Ministry of Research (BMBF) by a grant under the KMU-innovativ program UVMOPA (FKZ: 13N13022). A.V. acknowledges support by the Marie Curie ITN EID project "NOLOSS", grant 675745, and the Karlsruhe School of Optics and Photonics (KSOP). 


\section{REFERENCES}

[1] S. Partel, S. Zoppel, P. Hudek, A. Bich, U. Vogler, M. Hornung, and R. Völkel, "Contact and proximity lithography using 193nm Excimer laser in Mask Aligner,” Microelectron. Eng. 87, 936-939 (2010).

[2] L. Stürzebecher, F. Fuchs, U. D. Zeitner, and A. Tünnermann, "High-resolution proximity lithography for nanooptical components," Microelectron. Eng. 132, 120-134 (2015).

[3] R. Silver, T. Germer, R. Attota, B. M. Barnes, B. Bunday, J. Allgair, E. Marx, and J. Jun, "Fundamental limits of optical critical dimension metrology: a simulation study,” Proc. SPIE 6518, 1-17 (2007).

[4] R. Voelkel, U. Vogler, A. Bich, P. Pernet, K. J. Weible, M. Hornung, R. Zoberbier, E. Cullmann, L. Stuerzebecher, T. Harzendorf, and U. D. Zeitner, "Advanced mask aligner lithography: new illumination system," Opt. Express 18, 20968-20978 (2010).

[5] [For ARPES spectroscopy see e.g.: https://commons.wikimedia.org/wiki/File:ARPESgeneral.png]

[6] M. Scholz, D. Opalevs, P. Leisching, W. Kaenders, G. Wang, X. Wang, R. Li, and C. Chen, "1.3mW tunable and narrow-band continuous-wave light source at $191 \mathrm{~nm}$," Opt. Express 20, 18659-18664 (2012).

[7] M. Scholz, D. Opalevs, P. Leisching, W. Kaenders, G.Wang, X.Wang, R. Li, and C. Chen, "A bright continuouswave laser source at $193 \mathrm{~nm}, ”$ Appl. Phys. Lett. 103, 051114 (2013).

[8] U. Eismann, M. Scholz, T. Paasch-Colberg, and J. Stuhler, "Short, shorter, shortest: Diode lasers in the deep ultraviolet," LaserFocusWorld 52, (2016).

[9] E.A. Salehand and M.C. Teich, [Fundamentals of Photonics], John Wiley \& Sons, New Jersey, chapter 14.1 (1991).

[10] M. Mizuguchi, H. Hosono, H. Kawazoe, and T. Ogawa, "Color center formation and time-resolved photoluminescence for $\mathrm{ArF}$ excimer laser irradiation in $\mathrm{CaF}_{2}$ single crystals," Proc. SPIE 3424, 60-70 (1998).

[11]R. E. Schenker, L. Eichner, H. Vaidya, S. Vaidya, and W. G. Oldham, "Degradation of fused silica at $193 \mathrm{~nm}$ and 213 nm," Proc. SPIE 2440, 118-125 (1995).

[12]C. Chen, Y. Wang, Y. Xia, B. Wu, D. Tang, K. Wu, Z. Wenrong, L. Yu, and L. Mei, "New development of nonlinear optical crystals for the ultraviolet region with molecular engineering approach," J. Appl. Phys. 77, 2268 (1995).

[13]D. Tang, Y. Xia, B. Wu, and C. Chen, "Growth of a new UV nonlinear optical crystal: KBe2(BO3)F2," J. Cryst. Growth 222, 125-129 (2001).

[14]T. Kanai, X.Wang, S. Adachi, S.Watanabe, and C. Chen, "Watt-level tunable deep ultraviolet light source by a KBBF prism-coupled device," Opt. Express 17, 8696-8703 (2009).

[15] [For general specifications of the $266 \mathrm{~nm}$ TopWave system from TOPTICA photonics AG see: http://www.toptica.com/products/single-frequency-lasers/topwave-266/]

[16] [For general specifications of the DLC pro controller from TOPTICA photonics AG see: http://www.toptica.com/products/tunable-diode-lasers/laser-driving-electronics/dlc-pro/]

[17][For general specifications of the TA-FHG pro system from TOPTICA Photonics AG see: http://www.toptica.com/products/customized-solutions/193-nm-sub-mw/ and http://www.toptica.com/products/tunable-diode-lasers/frequency-converted-lasers/ta-fhg-pro/]

[18]R. Kirner, A. Vetter, D. Opalevs, C. Gilfert, M. Scholz, P. Leisching, T. Scharf, W. Noell, C. Rockstuhl, and R. Voelkel, "Mask-aligner lithography using a continuous-wave diode laser frequency-quadrupled to $193 \mathrm{~nm}$," Opt. Express 26, 730-743 (2018).

[19] A. Sahm, S. Baumgärtner, J. Hofmann, P. Leisching, and K. Paschke, "Miniaturized semiconductor MOPA laser source at $772 \mathrm{~nm}$ for the generation of UV laser light," Proc. SPIE 10535, 10535-72 (2018).

[20]S. Baumgärtner, S. Juhl, D. Opalevs, A. Sahm, J. Hofmann, P. Leisching, and K. Paschke, "Intensity noise properties of compact laser device based on miniaturized MOPA system for spectroscopic applications," Proc. SPIE 10513, 10513-8 (2018).

[21]H. Xuan, Z. Zhao, H. Igarashi, S. Ito, K. Kakizaki, and Y. Kobayashi, "300 mW narrow-linewidth deep-ultraviolet light generation at $193 \mathrm{~nm}$ by frequency mixing between Yb-hybrid and Er-fiber lasers," Opt. Express 23, 1056410572 (2015). 